\title{
The X-ray Variability of High-Redshift QSOs
}

\author{
J. Manners, O. Almaini, \& A. Lawrence \\ Institute for Astronomy, University of Edinburgh, Royal Observatory, \\ Blackford Hill, Edinburgh EH9 $3 H J$
}

\begin{abstract}
We present an analysis of X-ray variability in a sample of $\mathbf{1 5 6}$ radio quiet quasars taken from the ROSAT archive, covering a redshift range $0.1<z<4.1$. Through combining light curves in ensembles we are able to identify trends in variability amplitude with luminosity and with redshift. The decline in variability amplitude with luminosity identified in local AGN $(z<0.1)$ is confirmed out to $z=2$. There is tentative evidence for an increase in QSO X-ray variability amplitude towards high redshifts $(z>2)$ in the sense that QSOs of the same X-ray luminosity are more variable at $z>2$. The simplest explanation for this effect may be that high-redshift QSOs are accreting at a higher efficiency than local AGN.
\end{abstract}

\section{Introduction}

Rapid X-ray variability appears to be very common in AGN. Temporal power spectra show 'red noise' (i.e. more power at lower frequencies), with the form $P(f) \propto f^{-\alpha}$ where $\alpha \approx 1.5$ (Lawrence \& Papadakis 1993, Green et al 1993). Departures from a featureless power spectrum are rare. Some evidence for quasiperiodic oscillations has been observed in NGC 5548, NGC 4051 (Papadakis \& Lawrence 1993, 1995) and IRAS 18325-5926 (Iwasawa et al 1998), and in a handful of AGN a turnover has been seen at low frequencies (e.g. Edelson \& Nandra 1999). A high frequency cut-off would indicate the size of the emission region, although this cannot yet be distinguished from the noise for even the most well studied AGN.

Variability studies of local AGN $(z<0.1)$, over a fixed timescale, indicate that more luminous sources vary with a lower amplitude. This may be explained if more luminous sources are physically larger in size and are actually varying more slowly. Alternatively, they may contain more independently flaring regions and so have a genuinely lower amplitude. The slope of this correlation has been calculated in a number of papers using overlapping samples of local AGN. Lawrence \& Papadakis (1993) and Green et al (1993) analyzed samples of light curves from the EXOSAT database. The variability amplitude was found to vary with luminosity as $\sigma \propto L_{X}^{-\beta}$ with $\beta \approx 0.3$. The most comprehensive analysis of the variability-luminosity relation was carried out by Nandra et al, 1997 (hereafter N97) for 18 local Seyferts observed with the ASCA satellite. They find $\beta=0.355 \pm 0.015$. Whether this well-defined correlation applies to high-redshift QSOs is not so clear. Observations of distant QSOs are generally of low signal-to-noise and measurements of variability in individual objects are 
poorly defined. Almaini et al (2000) developed a technique to measure the amplitude of variability for low signal-to-noise sources and thus high-redshift AGN. By combining light curves from a number of AGN they were able to measure the amplitude of variability over ranges in luminosity and redshift. They studied a sample of 86 QSOs from the Deep ROSAT Survey of Shanks et al (1991) spanning a wide range in redshift $(0.1<z<3.2)$. The behaviour of variability amplitude with luminosity was found to be in rough agreement with the anti-correlation seen in local AGN but showing a possible upturn for the most luminous sources. Tentative evidence suggested this was due to increased variability at high redshifts, although a definite trend in the redshift behaviour could not be clearly confirmed.

In this paper we use the techniques of Almaini et al (2000) to determine the amplitude of variability in an expanded sample of QSOs taken from the ROSAT archive. QSOs at $z>1$ are preferentially selected in order to constrain the redshift behaviour of X-ray variability. A cosmology with $q_{0}=0.5, H_{0}=50 \mathrm{~km}$ $\mathrm{s}^{-1} \mathrm{Mpc}^{-1}$ is used throughout.

\section{QSO Sample \& Data Reduction}

The sample consists of 156 QSOs between $0.08<z<4.11$ taken from the ROSAT PSPC archive. It is made up of QSOs taken from a number of sources that all adhere to the following selection criteria: radio quiet quasar IDs, X-ray exposures $>10,000$ seconds, flux signal-to-noise $>5$, within 20 arcmin of the ROSAT pointing, and at least 3 time bins in the light curve.

The redshift distribution of the entire sample is displayed in Fig. 1. The data were obtained from the LEDAS online database facility at Leicester. Each source was extracted using a circular mask of radius chosen to include 90 per cent of the PSF. The data were then filtered to remove periods of high particle background and restrict the energy range to $0.1-2.4 \mathrm{keV}$. After careful background subtraction, an algorithm was used to construct light curves and bin up the data to allow meaningful Gaussian statistics.

The method used for measuring the amplitude of intrinsic variability is fully described in Almaini et al. (2000). To compare objects of different flux, the light curves are divided by the mean, so that measurements of fractional variability are made. A maximum likelihood technique is used to separate the intrinsic variations in the light curve from those due to noise. Individual measurements of variability were of low signal-to-noise, therefore in order to extract meaningful information from the sample it was necessary to combine the light curves into ensembles over given ranges of luminosity or redshift. Variability amplitudes are normalized to a timescale of 1 week, assuming the power spectrum slope seen in local AGN $(\alpha=1.5)$. Corrections are also made for time dilation and the effects of irregular binning.

\section{The Results}

Maximum likelihood estimates of the corrected intrinsic variability amplitudes were calculated for all 156 quasars. Nearly half the sample (69 QSOs) give a nonzero estimate of variability amplitude. The remaining QSOs can only provide 


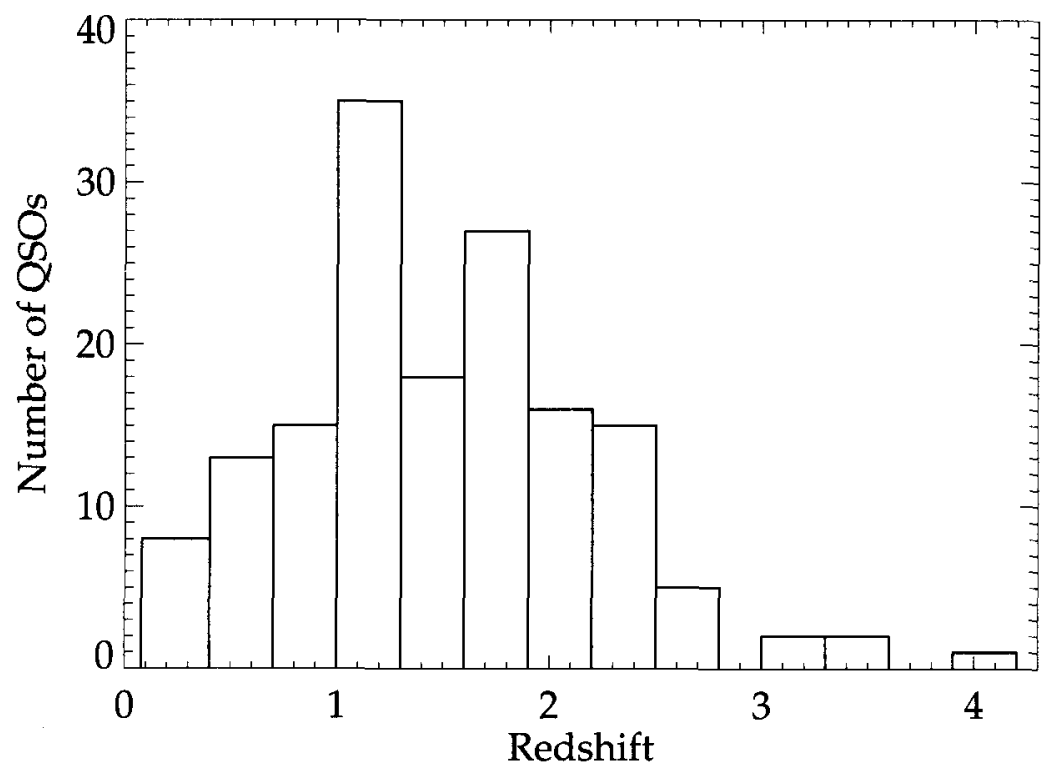

Figure 1. Redshift distribution of our sample of 156 RQQs.

upper limits, mainly due to low signal-to-noise. Treating the entire sample as one ensemble, the corrected mean ensemble variability is: $\sigma=0.15 \pm 0.01$ $(\sigma=0.16 \pm 0.01$ uncorrected) on a time-scale of 1 week.

The data were split into 6 luminosity bins each of $\sim 0.5 \mathrm{dex}$, and 11 redshift bins each of $\delta z=0.3$. For each ensemble, maximum likelihood estimates were calculated for the intrinsic variability amplitude in the combined light curve. Fig. 2 displays the ensemble results. Immediately apparent in Fig. 2(a) is an anti-correlation between quasar luminosity and variability amplitude. A power law fit to the individual quasars gives the best fit relation: $\sigma \propto L_{X}^{-\beta}$ where $\beta=0.18 \pm 0.05$ (plotted as a dot-dash line). This gives a fairly poor fit to the ensemble points, mainly due to some highly variable, high luminosity QSOs. However, a power law fit to QSOs with redshift less than 2 (solid line, $\beta=$ $0.27 \pm 0.05$ ) passes through the majority of the ensemble points and is very close to the average relation found for local AGN (plotted as a dashed line). The errors quoted here are 68 per cent confidence limits for the slope on allowing the normalization to float to its optimum value.

For the redshift dependence (Fig. 2b), it is reasonable to expect a certain amount of degeneracy with luminosity. This effect appears to dominate at low and medium redshifts. At redshifts beyond $\sim 2$, an upturn in variability is observed which cannot be explained as a consequence of the known trend with luminosity.

In order to decouple the effects of luminosity and redshift, the variability amplitude was plotted as a function of luminosity for 3 redshift intervals (Fig. 3). 

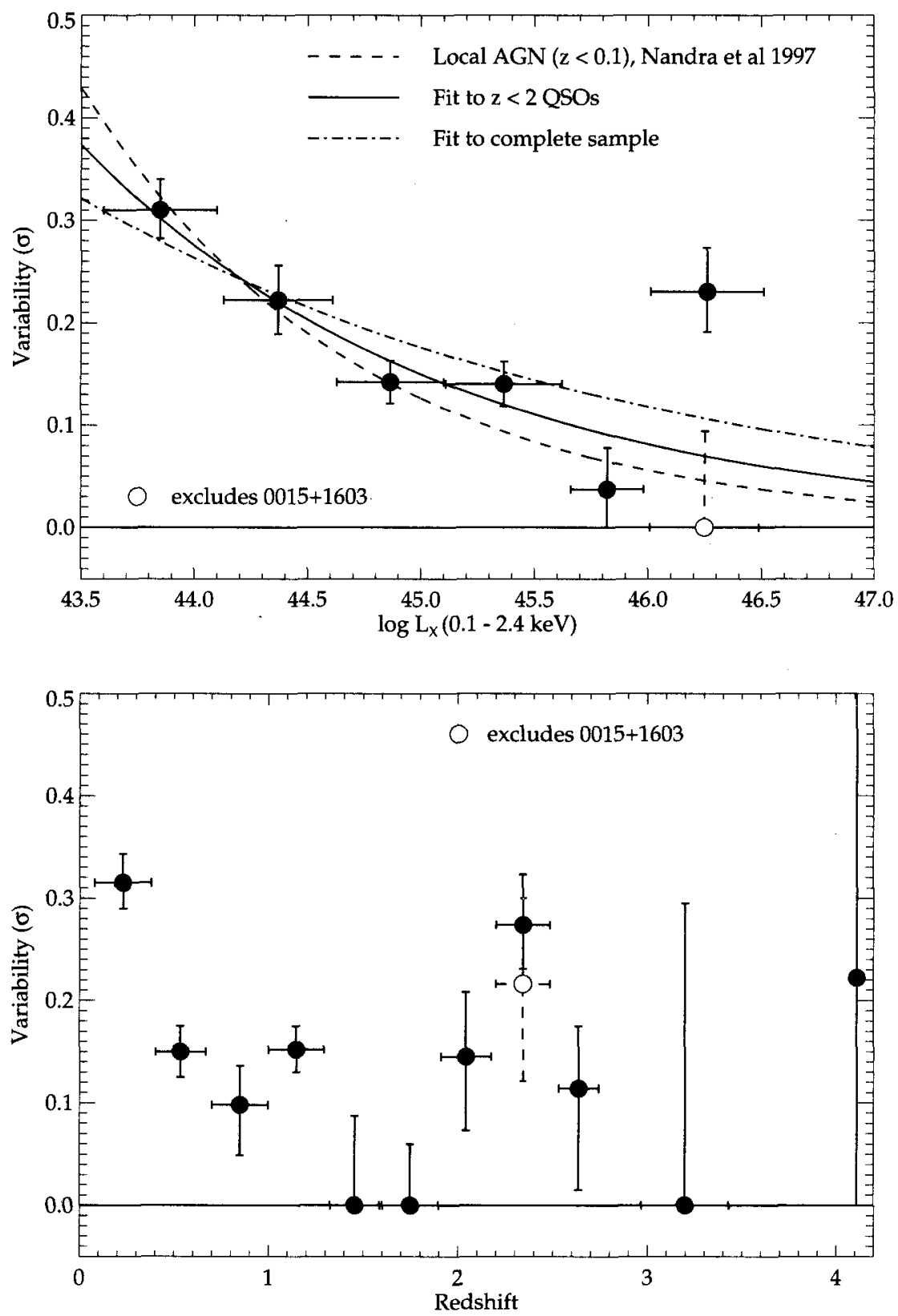

Figure 2. Variability amplitude as a function of luminosity (a) and redshift (b) for the 156 QSOs, displayed in ensemble form. The exclusion of QSO $0015+1603$ is explained in Section 4. 


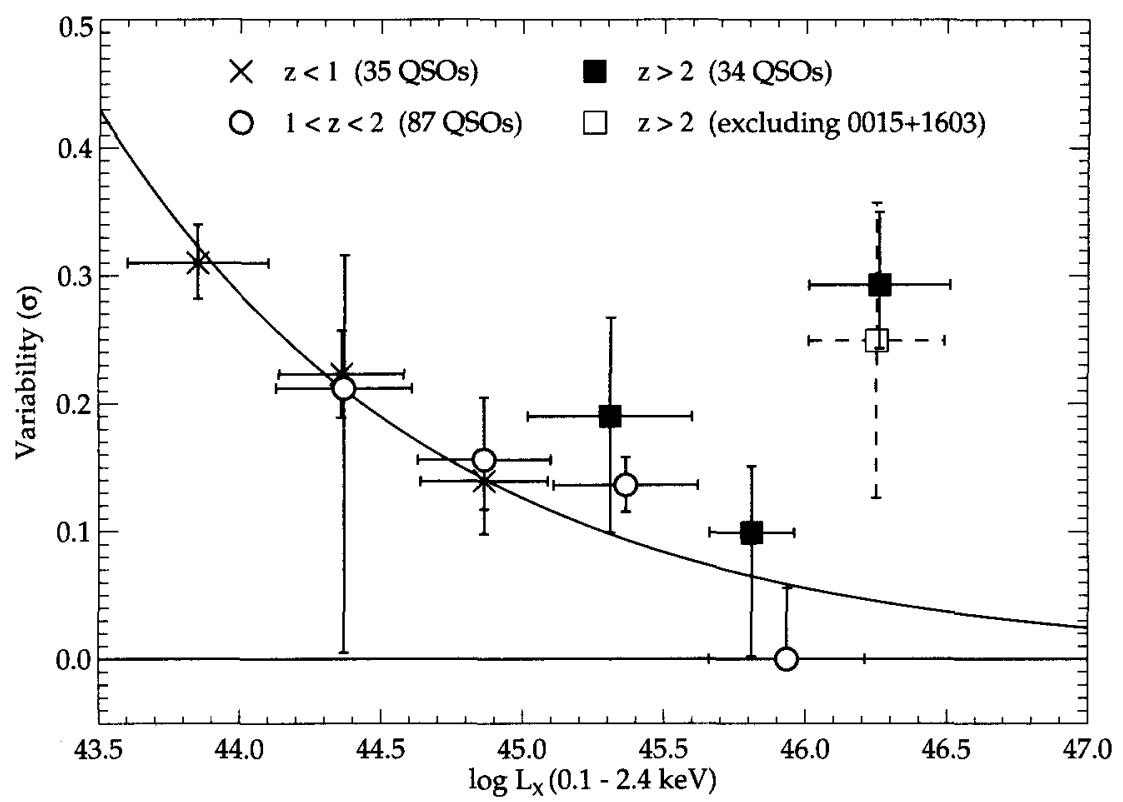

Figure 3. Variability amplitude as a function of luminosity over 3 redshift intervals. The line plotted is the best fit relation to local $(z<0.1)$ AGN found by Nandra et al (1997).

The luminosity bins for each redshift interval overlap, providing a measure of the change in variability amplitude as a function of redshift. Comparing the first 2 redshift intervals we find no significant difference in the $\sigma-L_{X}$ relation. The anti-correlation between variability and luminosity appears to be unchanged out to a redshift of 2 . To compare this with the relationship for local AGN, the power-law slope found by N97 is plotted on Fig. 3. For the redshift interval $z>2$ all 3 points show an 'excess' variability indicating that high-redshift QSOs may not be well characterized by the variability-luminosity correlation of local AGN.

\section{Properties of High-z Variable QSOs}

The apparent increase in variability seen in high redshift QSOs may be due to the inclusion of a new population of objects rather than differences in the 'typical' population. Narrow-line Seyfert 1s are known to exhibit enhanced variability (Boller et al 1996, Leighly 1999) and could be responsible for this upturn if their high-z equivalents were more prevalent than they are today. In order to test this hypothesis, the identifications for the 12 QSOs that exhibit detected variability at redshifts greater than 1.9 (i.e. the last 5 redshift intervals in Fig. $2 \mathrm{~b}$ ), were studied in more detail. All the QSOs with adequate optical spectra were found to contain broad permitted lines (> $3000 \mathrm{~km} \mathrm{~s}^{-1}$ FWHM). In one 
object, $0015+1603$, the optical grism spectrum was not of sufficient quality to determine linewidths. This object also displays a steep X-ray spectrum, so is a (potential) NLS1 candidate. $0015+1603$ was the highest luminosity object in the sample with a high significance detection of variability. To determine the effect of this steep-spectrum QSO on the characteristics of the sample, the variability analysis was repeated with the object removed. The affected bins are plotted as unfilled points in Figs. $2 \& 3$. Removing this highly variable object decreases the significance of the upturn in variability for the high-z sample, but does not affect the direction of the trend.

\section{Discussion}

There is still no definitive explanation for the anti-correlation between X-ray variability and luminosity. In particular, it is unclear whether variability is intrinsically linked with luminosity, or whether the link is to a third parameter that happens to scale with luminosity. If the upturn in variability observed at high redshifts is real, then an extra parameter must exist. X-ray variability timescale may directly scale with black hole mass if, for example, the emission occurs at a fixed number of Schwarzschild radii. This hypothesis has been supported by recent studies of Ptak et al (1998) and Iwasawa et al (2000). Low-luminosity AGN (LLAGN) that are expected to harbour relatively massive black holes display very little X-ray variability, while the dwarf Seyfert NGC 4395, which is thought to contain a small black hole, displays a large variability amplitude. If the amplitude of X-ray variability is linked to black hole mass, the upturn in variability seen at high redshifts would indicate that early quasars are undergoing far more efficient accretion than local AGN.

\section{References}

Almaini, O., Lawrence, A., Shanks, T., et al. 2000, MNRAS, 315, 325

Boller, T., Brandt, W.N., Fink, H. 1996, A\&A, 305, 53

Edelson, R., Nandra, K., 1999, ApJ, 514, 682

Green, A.R., McHardy, I.M., \& Lehto, H.J. 1993, MNRAS265, 664

Iwasawa, K., Fabian, A.C., Brandt, W.N., et al. 1998, MNRAS, 295, L20

Iwasawa, K., Fabian, A.C., Almaini, O., et al. 2000, MNRAS, 318, 879

Lawrence, A. \& Papadakis, I. 1993, A\&A, 414, L85

Leighly, K.M. 1999, ApJS, 125, 297

Nandra, K., George, I.M., Mushotzky, R.F., et al. 1997, ApJ, 476, 70

Papadakis, I.E. \& Lawrence, A. 1993, Nat, 361, 250

Papadakis, I.E. \& Lawrence, A. 1995, MNRAS, 272, 161

Ptak, A., Yaqoob, T., Mushotzky, R., et al. 1998, ApJ, 501, L37

Shanks, T., Georgantopoulos, I., Stewart, G.C., et al. 1991, Nat, 353, 315 